International Journal of Pure and Applied Mathematics

Volume 88 No. 3 2013, 381-390

ISSN: 1311-8080 (printed version); ISSN: 1314-3395 (on-line version)

url: http://www.ijpam.eu

doi: http://dx.doi.org/10.12732/ijpam.v88i3.6

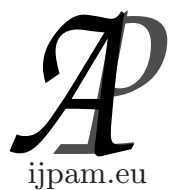

\title{
MODELLING THE EFFECT OF VACCINATION ON THE TRANSMISSION DYNAMICS OF MEASLES
}

\author{
A.A. Momoh ${ }^{1}$, M.O. Ibrahim ${ }^{2}$, I.J. Uwanta ${ }^{3}$, S.B. Manga ${ }^{4}$ \\ ${ }^{1}$ Department of Mathematics \\ Modibbo Adama University of Technology \\ Yola, Adamawa State, NIGERIA \\ ${ }^{2}$ Department of Mathematics \\ University of Ilorin \\ Ilorin, Kwara State, NIGERIA \\ ${ }^{3,4}$ Department of Mathematics \\ Usmanu Danfodiyo University \\ Sokoto, Sokoto State, NIGERIA
}

\begin{abstract}
Protecting children from vaccines-preventable diseases such as measles, is among primary goals of health administrators worldwide. Since vaccination turned out to be the most effective strategy against childhood disease, developing a framework that would predict an optimal vaccine coverage level needed to control the spread of these disease is crucial. In this paper, we used a compartmental mathematical model to study the transmission dynamics of measles. The effect of vaccination on transmission dynamics of measles were study. The stability of the disease-free equilibrium is established. Numerical simulations are carried out. We discussed in details the implications of our analytic and numerical solutions.
\end{abstract}

AMS Subject Classification: 92D40, 92D25, 34D20

Key Words: vaccination, endemic, equilibrium states, compartmental model, reproductive number

Received: June 25, 2013

(C) 2013 Academic Publications, Ltd. url: www.acadpubl.eu

${ }^{\S}$ Correspondence author 


\section{Introduction}

Worldwide, measles vaccination has been very effective, preventing an estimated 80 million cases and 4.5 million deaths annually [15]. Although, global incidence has been significantly reduced through vaccination, measles remains an important public health problem. Since vaccination coverage is not uniformly high worldwide, measles stands as the leading vaccine-preventable killer of children worldwide; measles is estimated to have caused 614,000 global deaths annually in 2002, with more than half of measles deaths occurring in Sub-Sahara Africa [14].

One disease whose mortality and morbidity burden has been dramatically reduced following large scale vaccination is measles [3]. New borns are afforded protection to measles through maternal antibodies, which may be effective for up to one year after birth. Vaccination against measles is not recommended until these antibodies have waned out. It has been demonstrated that vaccine efficacy is substantially higher in older infants with no maternal antibodies [5] and [9], hence current policy is to administer the MMR (measles-mumpsrubella) vaccine to infants between their first and second birthdays. While it seems that measles is mostly controlled in the US, Canada and other developed countries, it remains a major killer in the developing world [3], [10] and [12]. Although natural measles infection confer lifelong immunity [1] and Whittle et al. (1999), the assumption that measles vaccine also confers permanent protection has been reconsidered following outbreaks among high school and university students in the US in the 1980's, many of these students had been vaccinated 15-20 years prior to infection [12] and [4]. Clinical studies have proposed several mechanisms for the outbreak, the most likely candidates being vaccine failure in some individuals and subsequently lost of immunity after vaccination [10] and [11].

Mathematical models have widely been used to investigate the impact of a single-dose vaccination strategy on disease control [6] and Kribs and [7]. These models have discussed the effect of vaccination coverage, vaccine efficacy, and the warning rate of vaccines. The goal of this study is to provide a framework by developing a mathematical model for the transmission dynamics of measles. The organization of this paper is as follows: the model is developed in Section 2, the threshold condition for measles eradication is determine in Section 3, numerical solution is presented in Section 4. The paper ends with a discussion section. 


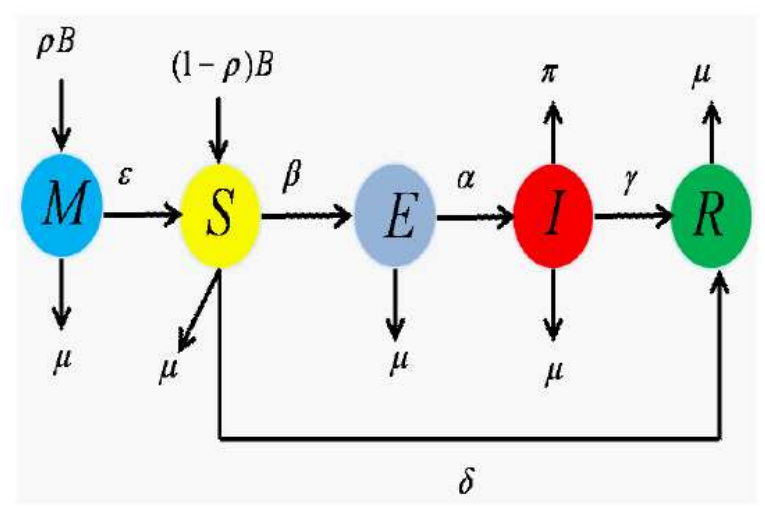

Figure 1: Transfer diagram of the model

\section{Model Derivation}

In this model, the total population $(\mathrm{N})$ is divided into five classes: Passively immuned infants (M), Susceptible (S), Exposed (E), Infected (I) and Recovered $(\mathrm{R})$. The class $\mathrm{M}$ of the passively immuned individuals increases due to inflow of proportion $\rho B$ of infants with passive immunization. This passive immunity may be due to the presence of material antibodies which interfere with vaccine-induced seroconversion and diminished by natural death at a rate $\mu$. The primary vaccine may wane with time and thus the passively immuned individuals gradually become fully susceptible to the disease again at a rate $\varepsilon$. The class $\mathrm{S}$ of susceptible individuals is generated through the inflow of the remaining individuals $(1-\rho B)$ who are not passively immuned at birth and also increases due to inflow of individuals from the passively immuned class $M$ due to waning out of the efficacy of vaccines at a rate $\varepsilon$. The susceptible class $\mathrm{S}$ is decreases by infection following contact with infected individuals at rate $\beta$ and vaccination at a rate $\delta$. the class S diminished by natural death at a rate $\mu$. The class $\mathrm{E}$ of the exposed individuals is generated through contact with infected individuals at a rate $\beta$, the class $\mathrm{E}$ is decreased by breakthrough into infected class at a rate and diminished by natural death at a rate $\mu$. The class I of infected individuals is generated by the breakthrough of exposed individuals into the infected class at a rate $\alpha$. The class is decreased by recovery from infection at a rate $\gamma$ and diminished by natural death at a rate $\mu$ and death as a result of measles infection at rate $\pi$. The model assumes that both vaccinated susceptible individuals and recovered infected individuals become permanently immuned to the disease. The class $\mathrm{R}$ of individuals who have complete protec- 
tion against the disease. The class $\mathrm{R}$ of immuned individuals is increased by the inflow of individuals from the susceptible class $\mathrm{S}$ at a rate $\delta$ and infected class I due to measles therapy at a rate $\gamma$. The class $\mathrm{R}$ of recovered individuals diminished by natural death at a rate $\mu$.

The transition between model classes can now be expressed by the following differential equations

$$
\begin{gathered}
\frac{d M}{d t}=\rho B-(\varepsilon+\mu) M, \\
\frac{d S}{d t}=(1-\rho) \mathrm{B}-(\mu+\delta) \mathrm{S}-\beta \mathrm{SI}+\varepsilon \mathrm{M}, \\
\frac{d E}{d t}=\beta \mathrm{SI}-(\mu+\alpha) \mathrm{E}, \\
\frac{d I}{d t}=\alpha \mathrm{E}-(\mu+\pi+\gamma) \mathrm{I}, \\
\frac{d R}{d t}=\gamma \mathrm{I}+\delta \mathrm{S}-\mu \mathrm{R} .
\end{gathered}
$$

A description of all the parameters with their estimated values is given in Table 1

\section{Disease Eradication}

In this section, the model is analyzed for its disease free equilibrium in order to provide the threshold condition for measle control or eradication. We note that the equation for the total population is $\frac{d N}{d t}=B-\mu N$. as $\mathrm{t} \longrightarrow \infty$, and hence $\mathrm{R}=\frac{B}{\mu}-M-S-E-I$. This shows that the feasible reqion

$$
\Omega=\left\{(M, S, E, I, R): M, S, E, I, R \geq 0, M+S+E+I+R=\frac{B}{\mu}\right\}
$$

is a positively invariant set for the model. Therefore, we restrict our attention to the dynamics of the model in $\Omega$. 
B Recruitment rate of individuals

$\rho$ Fraction of recruited individuals who receive 0.25 vaccine

$\varepsilon \quad$ Rate at which maternal antibodies wanes out $\quad 0.2$

$\mu \quad$ Natural death rate $\quad 0.2$

$\alpha$ Rate at which exposed individuals become in- 0.1 fected

$\gamma \quad$ Rate at which infected individuals become re- 0.2 covered

$\delta \quad$ Rate at which susceptible become immuneddue o.52,0.595,0.68,0.769 to vaccination

$\beta$ Contact rate $\quad 0.01$

$\pi \quad$ Infection induced death $\quad 0.02$

Table 1: Description and estimation of the model parameters

\subsection{Disease Free Equilibrium (DFE)}

In the absence of infection, the model has a unique disease free equilibrium $\mathrm{P}_{0}=\left(M^{0}, S^{0}, E^{0}, I^{0}, R^{0}\right)$ where

$$
M^{0}=\frac{\rho B}{(\varepsilon+\mu)}, \quad \mathrm{S}^{0}=\frac{B(\varepsilon+\mu-\mu \rho)}{(\varepsilon+\mu)(\mu+\delta)}, \quad \mathrm{R}^{0}=\frac{\delta B(\varepsilon+\mu-\mu \rho)}{\mu(\varepsilon+\mu)(\mu+\delta)} .
$$

To analyse the stability of the DFE, the model (1)-(5) is linearized around $\mathrm{P}_{0}$ we evaluate the Jacobian matrix at $\mathrm{P}_{0}=\left(M^{0}, S^{0}, E^{0}, I^{0}, R^{0}\right)$

Thus

$$
\begin{aligned}
& J\left(P_{0}\right)=\left(\begin{array}{ccccc}
-(\mu+\varepsilon) & 0 & 0 & 0 & 0 \\
\varepsilon & -(\mu+\delta) & 0 & \frac{-\beta B(\varepsilon+\mu-\mu \rho)}{(\varepsilon+\mu)(\mu+\delta)} & 0 \\
0 & 0 & -(\mu+\alpha) & \frac{-\beta B(\varepsilon+\mu-\mu \rho)}{(\varepsilon+\mu)(\mu+\delta)} & 0 \\
0 & 0 & \alpha & -(\mu+\pi+\gamma) & 0 \\
0 & \delta & 0 & \gamma & -\mu
\end{array}\right), \\
& \left|J\left(P_{0}\right)-\lambda I\right| \\
& =\left|\begin{array}{ccccc}
-(\mu+\varepsilon)-\lambda & 0 & 0 & 0 & 0 \\
\varepsilon & -(\mu+\delta)-\lambda & 0 & \frac{-\beta B(\varepsilon+\mu-\mu \rho)}{(\varepsilon+\mu)(\mu+\delta)} & 0 \\
0 & 0 & -(\mu+\alpha)-\lambda & \frac{\beta B(\varepsilon+\mu-\mu \rho)}{(\varepsilon+\mu)(\mu+\delta)} & 0 \\
0 & 0 & \alpha & -(\mu+\pi+\gamma)-\lambda & 0 \\
0 & \delta & 0 & \gamma & -\mu-\lambda
\end{array}\right|
\end{aligned}
$$


We have the following stability result that shows $R_{0}$ is a sharp threshold.

Proposition 1. $P_{0}$ is locally asymptotically stable if $R_{e}(\lambda)<0$.

Proof. Three eigenvalues of $\mathrm{J}\left(\mathrm{P}_{0}\right)$ are $\lambda_{1}=-\mu, \lambda_{2}=-(\mu+\delta)$ and $\lambda_{3}=$ $-(\mu+\varepsilon)$. The other two eigenvalues $\lambda_{4}, \lambda_{5}$ are eigenvalues of $2 \times 2$ matrix.

Let

$$
\left(\begin{array}{cc}
-(\mu+\alpha) & \frac{\beta B(\varepsilon+\mu-\mu \rho)}{(\varepsilon+\mu)(\mu+\delta)} \\
\alpha & -(\mu+\pi+\gamma)
\end{array}\right) .
$$

We want to show that the Routh-Hurwitz conditions holds, namely, $\operatorname{tr}(A)<0$ and $\operatorname{det}(A)>0$. Simple calculation show that $\operatorname{tr}(\mathrm{A})=-(\mu+\alpha)-(\mu+\pi+\gamma)$. It appears that $\operatorname{tr}(A)<0$ since all the parameters are non negative.

$$
\operatorname{det}(\mathrm{A})=(\mu+\alpha)(\mu+\pi+\gamma)-\frac{\beta B \alpha(\varepsilon+\mu-\mu \rho)}{(\varepsilon+\mu)(\mu+\delta)} .
$$

Setting $\operatorname{det}(A)>0$ we have,

$$
\begin{gathered}
(\mu+\alpha)(\mu+\pi+\gamma)-\frac{\beta B \alpha(\varepsilon+\mu-\mu \rho)}{(\varepsilon+\mu)(\mu+\delta)}>0, \\
(\mu+\alpha)(\mu+\pi+\gamma)>\frac{\beta B \alpha(\varepsilon+\mu-\mu \rho)}{(\varepsilon+\mu)(\mu+\delta)} .
\end{gathered}
$$

Thus $\operatorname{det}(A)>0$ if $(\mu+\alpha)(\mu+\pi+\gamma)>\frac{\beta B \alpha(\varepsilon+\mu-\mu \rho)}{(\varepsilon+\mu)(\mu+\delta)}$.

This proves the proposition.

\section{Numerical Solution and Results}

We carry out numerical simulations of our model (1)-(5) in a hypothetical population of size 1000 . We will vary key parameters to investigate the impact of vaccination on the transmission dynamics of measles. We have estimated the values of our model parameters as presented in Table 1 .

In Figure 2, we fixed the vaccination coverage rate at $60 \%$ where the vaccine has a success rate of $85 \%$. This means that $\delta=0.51$. 


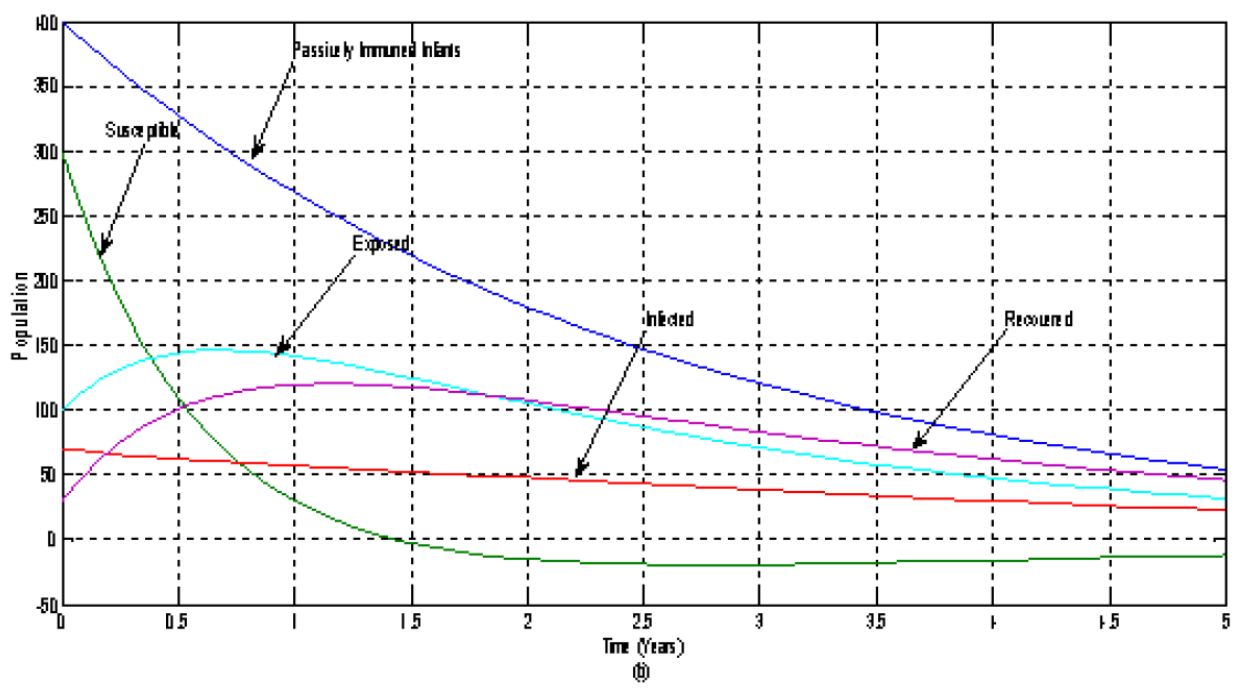

Figure 2: Simulation result showing the impact vaccination on measles transmission. In (a), $\mathrm{B}=0.32, \rho=0.25, \varepsilon=0.2, \mu=0.2, \alpha=0.1, \gamma=$ $0.2, \beta=0.01, \pi=0.02$.

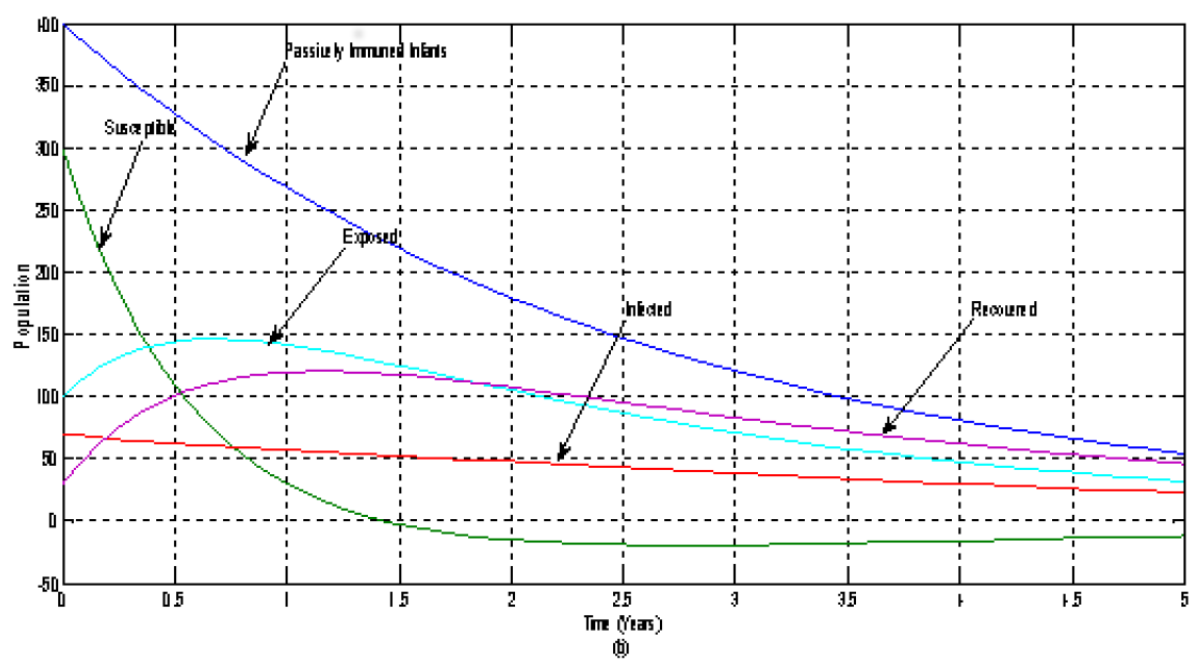

Figure 3: Simulation result showing the impact vaccination on measles transmission. In (a), $\mathrm{B}=0.32, \rho=0.25, \varepsilon=0.2, \mu=0.2, \alpha=0.1, \gamma=$ $0.2, \beta=0.01, \pi=0.02$.

In Figure 3, we fixed the vaccination coverage rate at $70 \%$ where the vaccine 
has a success rate of $85 \%$. This means that $\delta=0.595$.

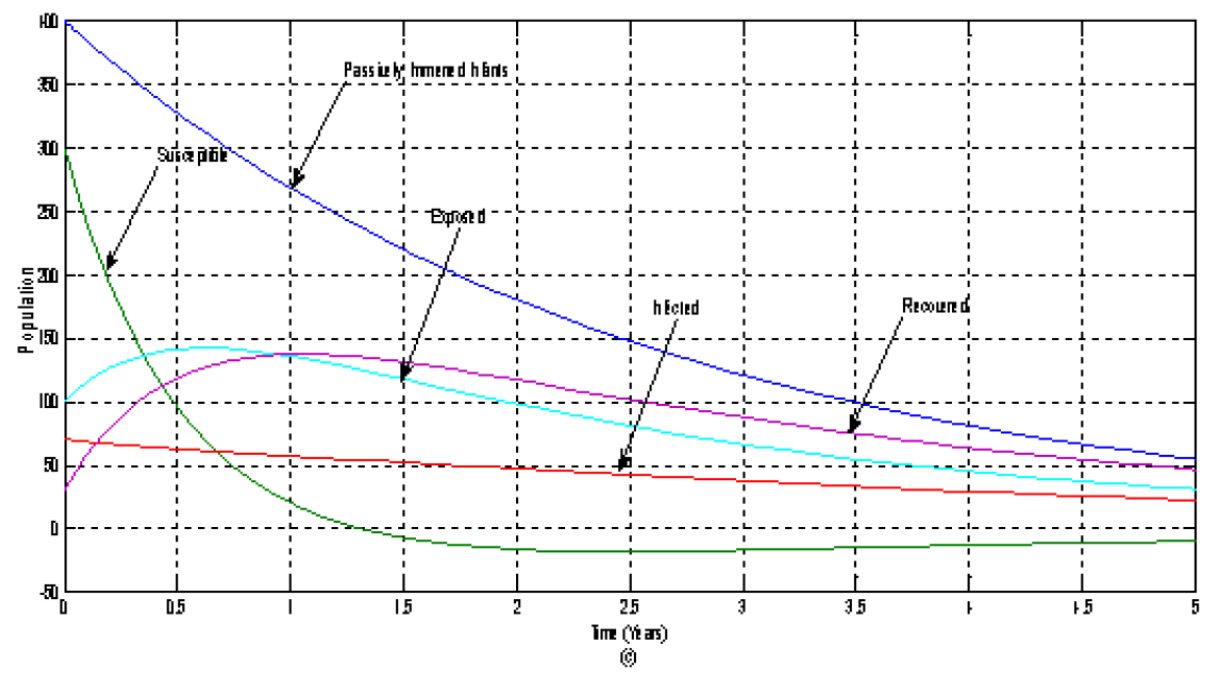

Figure 4: Simulation result showing the impact vaccination on measles transmission. In (a), $\mathrm{B}=0.32, \rho=0.25, \varepsilon=0.2, \mu=0.2, \alpha=0.1, \gamma=$ $0.2, \beta=0.01, \pi=0.02$.

In Figure 4, we fixed the vaccination coverage rate at $80 \%$ where the vaccine has a success rate of $85 \%$. This means that $\delta=0.680$.

In Figure 5, we fixed the vaccination coverage rate at $90 \%$ where the vaccine has a success rate of $85 \%$. This means that $\delta=0.769$.

\section{Discussion}

We see in figure 2 that, if only $60 \%$ of the susceptible individuals are vaccinated against measles, the number of passively immuned infants and susceptible individuals decrease, significantly, but the number of exposed individuals and infected individuals are still high. This is not a desirable result as it is the exposed individuals that become infectious with time. The recovered individuals increase slightly. If we increase the vaccination rates from $60 \%$ to $70 \%, 80 \%$ and $90 \%$ respectively maintaining the success rate at $85 \%$ as represented in figures 3,4 and 5, a more dramatic change occurs in the disease dynamics. The number exposed individuals show a much greater decline while the numbers of infected individuals remain low. This demonstrates that high vaccination coverage can be an effective control measure in high measles prevalence countries. 


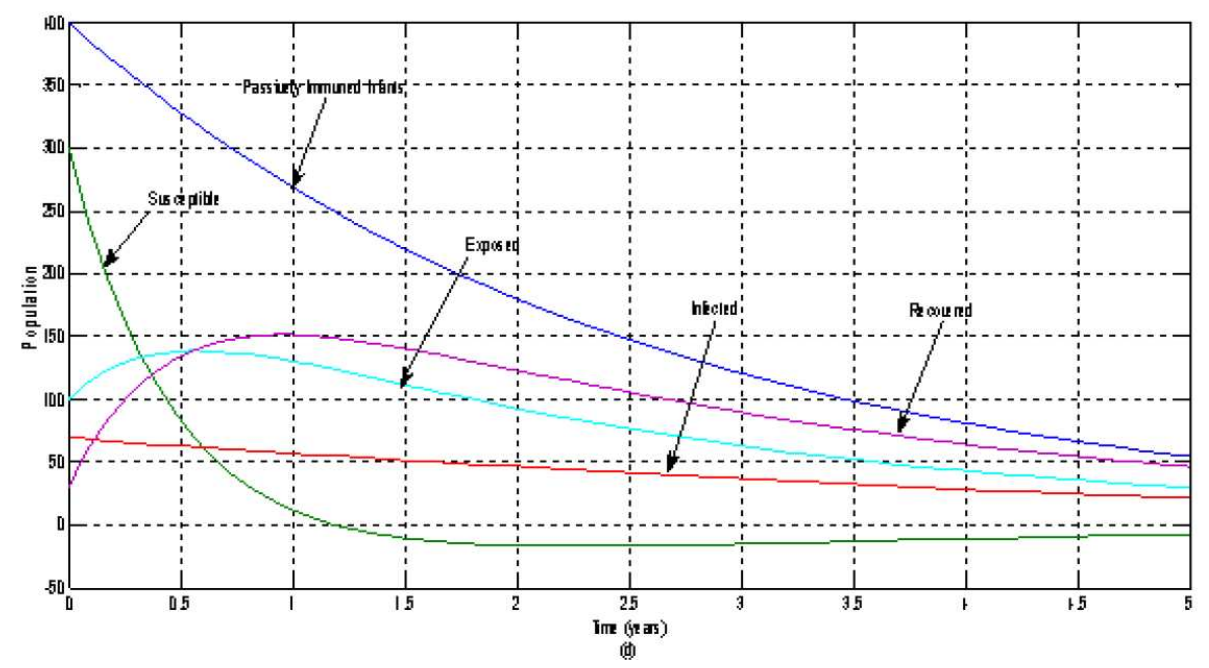

Figure 5: Simulation result showing the impact vaccination on measles transmission. In (a), $\mathrm{B}=0.32, \rho=0.25, \varepsilon=0.2, \mu=0.2, \alpha=0.1, \gamma=$ $0.2, \beta=0.01, \pi=0.02$.

\section{Conclusion}

Comparing our simulation results in Figures 2, 3, 4 and 5, we conclude that, in high measles prevalence countries, effective vaccination will have a greater impact on the transmission dynamics of the disease.

\section{References}

[1] R.E. Behrman, R.M. Kliegman, Nelson Essentials of Paediatrics, Saunders (1998).

[2] R.T. Chen, L.E. Markowitz, P. Albrecht, Measles antibodies: Reevaluation of protective titres, J. Infect. Dis., 162 (1990) 1036-1062.

[3] M.A. Garly, P. Aaby, The challenge of improving the efficacy of measles vaccine, Acta Tropica, 85 (2003), 1-17.

[4] T.L. Gustavson, A.W. Lievens, P.A. Brunell, Measles outbreak in a fully immunized secondary school population, 316, (1987) 771-774.

[5] W. Janaszek, J. Slusarczyk, Immunity against measles in population of women and infants in Poland, Vaccine 21, 2948-2953 (2003). 
[6] C.M. Kribs-Zaleta, M. Martcheva, Vaccination strategies and backward bifurcation in an age-since-infection structured model, Math. Biosci., 177 (2002), 317-332.

[7] C.M. Kribs-Zaleta, J.X. Velasco-Hern'andez, Asimple vaccination model with multiple endemic states, Math. Biosci., 164 (2000), 183-201.

[8] A.R. McLean, R.M. Anderson, Measles in developing countries, Part I: Epidemiological parameters and patterns, Epidemiol. Infect., 100 (1988), $11-133$.

[9] J. Mossong, C.P. Muller, Modelling measles re-emergence as a result of waning of immunity in vaccinated population, Vaccine, 21 (2003), 45974603.

[10] J. Mossong, J. Nokes, D.J. Edmunds, W.J. Cox, M.J. Ratman, C.P. Muller, Modelling the impact of subclinical measles transmission in vaccinated populations with waning immunity, Am. J. Epidemiol., 150 (1999), 12381249.

[11] B. Shulgin, L. Stone, A. Agur, Pulse vaccination strategy in the SIR epidemic model, Bull. Math. Biol., 60 (1998), 1123-1148.

[12] H.C. Whittle, P. Aaby, B. Samb, H. Jensen, J. Bennet, F. Simondon, Effect of subclinical infection on maintaining immunity against measles in vaccinated children in West Africa, Lancet, 353 (1999), 98-102.

[13] World Health Organization. Measles vaccines: WHO position paper, Wkly. Epidemiol. Rec., 79 (2008), 130-142.

[14] World Health Organization, Department of Vaccines and Biologicals, 2001. Measles Technical Working Group: strategies for measles control and elimination. Report of a meeting, Geneva, 11-12 May 2000. Geneva, Switzerland: World Health Organization. 